\title{
Near-Death Experiences in India: They Have Tunnels Too
}

\author{
Susan J. Blackmore, Ph.D. \\ University of the West of England
}

ABSTRACT: An advertisement in an Indian newspaper solicited accounts from people who had come close to death. Of 19 received, 7 reported no experiences, 4 reported dreamlike experiences, and 8 reported near-death experiences (NDEs). These NDEs were comparable to those reported by Raymond Moody, and included tunnels, dark spaces, and bright lights, contrary to previous reports of Indian cases. Many respondents reported positive life changes regardless of whether or not they had an NDE.

It is important to know whether near-death experiences (NDEs) are consistent across cultures, although the evidence at present is very limited. The largest cross-cultural study was carried out by Karlis Osis and Erlendur Haraldsson (1977), who sent out questionnaires to 5,000 doctors and nurses in the United States and interviewed 704 medical personnel in India. They inquired not only about NDEs but about the medical workers' recollections of the experiences told to them by people who were dying. These accounts were secondhand and often told many years after the events. Nonetheless the findings showed that the most common visions were of dead people or religious figures, to a large extent dependent upon the person's religious background. Often the figures seemed to have come to take the person away, and while the Americans were usually willing to go, the Indians more often refused.

Susan J. Blackmore, Ph.D., is Senior Lecturer in the Department of Psychology at the University of the West of England. Reprint requests should be addressed to Dr. Blackmore at the Department of Psychology, University of the West of England, St. Matthias College, Bristol BS16 2JP, England. 
Although these were accounts from people who did die, they were similar to the few accounts of NDEs in India. Satwant Pasricha and Ian Stevenson (1986) reported 16 cases of NDEs in India, and a common theme was the story of mistaken identity. Religious figures came to take the person away, but then discovered that it was the wrong person. Their cases seemed dramatically different from the type of NDE first described by Raymond Moody (1975). There were no tunnels, no bright lights, and no out-of-body experiences (OBEs), in which the experiencers see their own bodies from a distance. This discrepancy might be used to argue against the universality of NDEs, and is particularly important for physiological theories.

Some researchers argue that all the features of the NDE can be accounted for in terms of the processes going on in the dying brain, or in the brains of those subjected to severe stress or fear of death. For example, Juan Saavedra-Aguilar and Juan Gómez-Jeria (1989) have presented a neurobiological model of the NDE in which they account for all the features of the NDE in terms of the effects of hypoxia, temporal lobe and limbic system dysfunction, and an imbalance in neurotransmitters. The temporal lobe and limbic system are closely related and have often been implicated in the NDE. For example, direct electrical stimulation of the temporal lobe can give rise to memory flashbacks (Penfield, 1955), and people with many signs of limbic lobe lability are also more prone to religious, psychic, and mystical experiences (Persinger, 1983).

Various neurotransmitters and neuromodulators have been implicated in NDEs. For example, Daniel Carr (1982) was the first to suggest that endogenous opiates, or endorphins, would be released during the stress of a near-death event and could account for the peaceful and joyful feelings. Moreover, endorphins lower the threshold for seizure activity in the limbic system and temporal lobe, and so can trigger the kind of limbic lobe syndrome often compared to NDEs. On the other hand, Melvin Morse, David Venecia, and Jerrold Milstein (1989) have argued that serotonin mechanisms are more likely to provide an explanation than endorphins.

None of these theories directly addresses the origin of the tunnel. In his early study of 102 people who had come close to death, Kenneth Ring (1980) found that 23 percent had "entered the darkness" and 16 percent saw the light. These experiences are often equated with the more obvious tunnels in which people describe flying through dark spaces, or even through pipes or tubes. Tom Troscianko and I have argued that the tunnel can be produced by any mechanism that causes disinhibition and consequent random firing in the cortex (Blackmore and Troscianko, 1989). 
The way cells in the visual cortex are organized, with many more cells devoted to the central area of the visual field, means that random firing would produce a much brighter impression in the center of the field of view, fading out towards the periphery: in other words, a tunnel pattern. This could be overlaid on, or mixed with, ongoing imagery to produce the complex tunnel experiences found in NDEs. Anoxia could be one of the triggers to produce such inhibition. There might be variation in the imagery overlaid on the tunnel, but the basic tunnel form should be equally common in all cultures regardless of beliefs or religious background.

The universality of the tunnel experience has been challenged by what little cross-cultural evidence there is. Pasricha and Stevenson (1986) found no tunnels among their 16 Indian cases, nor among further cases still being investigated (I. Stevenson, personal communication, 1992). If this lack of tunnels in a different culture were confirmed, it would provide evidence against the physiological theories. I should point out that some alternative nonphysiological theories also predict that tunnels should be widespread. For example, Ring's (1980) holographic theory might predict that everyone should see the light of the hologram. Theories that equate the NDE with reliving birth might predict that everyone should re-experience traveling down the birth canal. However, if features such as tunnels and lights were not found in different cultures, those theories might still survive, since their predictions are not very clear. By contrast, the physiological theories make a much clearer prediction, and evidence of a cultural bias in tunnels would be evidence against them.

For these reasons I wanted to collect accounts of near-death encounters from India. The aim was to solicit accounts from people who had come close to death, without suggesting what experiences I was interested in, and to see whether any people spontaneously described tunnels or bright lights.

This study was not designed to explore other aspects of the NDE, but in fact many respondents described in great detail the effects their experiences had on them. The aftereffects of NDEs have been well documented (Grey, 1985; Lorimer, 1990; Ring, 1984). Generally NDErs report that their fear of death is reduced or abolished altogether, an effect that was reported even before Moody's work popularized the NDE (Dobson, Tattersfield, Adler, and McNicol, 1971; Druss and Kornfeld, 1967). More recently Charles Flynn (1982) surveyed 21 NDErs and found that an increase in concern for others was the most consistent effect.

However, it is becoming increasingly clear that you do not need to have an NDE to experience these changes. Having similar experi- 
ences, such as OBEs (Gabbard and Twemlow, 1984) or encounters with unidentified flying objects (Davis, 1988; Ring, 1992), can have comparable effects, as can coming close to death but without an NDE (Ring, 1980). Therefore it made sense to compare the aftereffects in this sample with those from previous reports.

\section{Method}

On November 2, 1991, I placed an advertisement in the Times of India headed: "Have you ever had a close brush with death?" I asked those who had, to write and tell me about the circumstances of their close brush with death, their experiences at the time, and any effects it had on them afterwards. I did not mention near-death experiences or tunnels, nor did I give any further idea of what was required.

A follow-up questionnaire was sent to all those who replied, asking for further details. The questionnaire included questions about the circumstances, any medication or resuscitation received, and emotional feelings at the time. I did not wish to use one of the standard NDE questionnaires (e.g., Greyson, 1983), because these might too readily suggest to respondents what they ought to be describing. I therefore included other questions addressed both to common features of NDEs and to other possible features, so as not to imply that there was anything special about the tunnel. Questions included:

Did you see any lights? What color were they?

Did you go into the light?

Did you see a doorway or gate?

Did you see a tunnel?

Did you see any regular patterns?

Did you have any hallucinations of any kind?

Other questions asked about OBEs, distant travel and vision, the decision to return, and changes in belief.

\section{Results}

I received 19 replies. Some were many pages long and included detailed descriptions and even photographs of the people concerned. Nine subsequently returned the questionnaire. From the accounts in the letters and the responses to the questionnaire, I was able to assign 
a score using Ring's (1980) Weighted Core Experience Index (WCEI). There are limitations to the accuracy I could achieve with the information available and it is possible that some respondents would achieve higher scores if I were able to interview them. Scores are therefore more likely to be underestimates than overestimates. I divided the respondents into three groups: (a) those who scored 0 on the WCEI and described no experience at all; (b) those who scored 0 on the WCEI but described dreamlike experiences of various kinds; and (c) those who achieved some score on the WCEI and therefore had at least part of the core experience.

\section{Nonexperiencers}

Seven respondents ( 37 percent) reported no experiences at the time of their close brush with death. These were involved in a car accident, a plane crash, a near-miss with a train, fear of having cancer, postoperative complications, a near-drowning, and an unexplained collapse. They described, sometimes in great detail, the circumstances of the events, but gave no hint of any experiences occurring at the time. Many described the effects that their close brush with death had on their life and beliefs, as described below.

\section{Hallucinatory Experiencers}

Four respondents (21 percent) reported experiences that were quite unlike the type of NDE described initially by Moody (1975). They scored 0 on Ring's WCEI because they included none of the classic components. These were strange dreamlike or hallucinatory experiences: a vision during a three-day coma of being with a mother hen and her chicks; a sensation, during complications after bypass surgery, of the thumb repeatedly bending into the palm of the hand; a vision during a near-drowning of watching a lake from the top of a tree; and a gruesome fight, during another drowning, with a 7-foot monster.

In this last case, an 80-year-old man described an experience that happened 70 years previously. When he was a young boy, he nearly drowned while his mother was washing clothes in the river. He wrote:

I felt that I was seated in a chariot speeding very rapidly towards a huge fellow seven foot high. He tried to run towards me and grab me by the neck but I was prepared to attack him. 
He went on to describe the fight between himself and death, as his mother tried to pull him out of the water. This experience still affects him after 70 years, and he also wrote about his recent dreams of death and flying to a golden city. This was not a typical NDE but did have the sensation of fast travel; however, travel without seeing or entering darkness obtains no points on the WCEI. I report this experience because I do not wish to select only those that were clearly Moody-type NDEs.

\section{Near-Death Experiencers}

Eight respondents (42 percent) reported at least some elements of the classic NDE and obtained some points on Ring's WCEI. These had nearly died through cardiac arrest (two respondents), electrocution, unexplained unconsciousness, heart surgery, coma and fever, a bus accident, and childbirth.

In their initial letters, only six reported NDE-like experiences. Although very varied, these were clearly Moody-type NDEs, and most included elements at least comparable to the classic idea of a tunnel.

Two further cases with NDE elements emerged from the questionnaires, although these had minimal experiences. One who had been involved in a bus accident and described extreme apprehension and fear reported on the questionnaire "visual hallucinations of darkness alternating with bright flashes," receiving 2 points on Ring's WCEI. Another who had nearly died in childbirth reported feeling that she might die at any moment and seeing "smugged" (smudged?) lights, receiving 3 points on the WCEI.

The six clearly Moody-type NDEs were as follows:

$N D E$ \#1. A 50-year-old woman described an experience that happened to her 18 years ago when, because of "tension on her mind," she fell down unconscious for a few minutes. She heard her maidservant and husband come running and calling her as if from a far distance:

At the same time I felt I was going through complete blackness and there was a tingling sound of tiny bells in my ears. The feeling was of complete relief and lightness. It was not at all a feeling of deep slumber, when we don't feel anything. As I said I was feeling ecstasy if that is the right word.

After this experience, which rated a score of 4 on the WCEI, she felt that her life was changed. She became more bold and learned to take 
life as it comes. She also stated: "I am no more afraid of death." She added that she had never read any books or heard that this kind of thing could happen, and only later learned that she was not the only one who had experienced it. She wondered whether it was the thought of her 4-year-old son that brought her back to life.

Her final comments could have come straight from Moody's (1975) original account of the NDE: "This is the first time I am writing to you openly, thinking you might believe. I tried to talk before but people think it is my imagination so I stopped talking." On the questionnaire she said she experienced a tunnel and described it as "very dark." To the question about regular patterns she wrote: "circles in spite of darkness." These circles were in the tunnel.

NDE \#2. A 72-year-old man wrote about his experience during angiography associated with a bypass operation:

I was in ICU and was just coming to. I suddenly got a feeling that someone was calling me away-almost saying "now you have to go"that your time "here" is over. I cannot quite describe it-it was such a confused experience, but definitely I thought 4 or 5 figures were beckoning me to "go away" from this world.

He wrote that he kept thinking about this experience, which rated a WCEI score of 4 , for some time, but it did not change his life in any way.

$N D E$ \#3. A 39-year-old man suffering from severe palpitations twice came close to death. He recalled no experiences from the time of his first brush with death. Six years later he passed out while playing badminton and had to be admitted to the intensive care unit (ICU) of a large general hospital. He wrote:

Before I was taken to hospital, something happened. I became unconscious and during this stage, I seemed to be floating in a dark space. I distinctly remember having an unusual peace of mind state for some brief moments. I felt totally at peace. I became conscious after a few minutes.

In the hospital following this experience, which rated a WCEI score of 6 , he had to be cardioverted with a direct current electrical shock of 100 watt-seconds. Seven years later he was tempted to play badminton again and again had the same experience and was resuscitated. $\mathrm{He}$ 
added that somehow he regretted leaving this mind state when he became conscious.

$N D E$ \#4. A 62-year-old man related events occurring when he was 13 years old. He had a high fever and cough and was in a coma for six to seven hours. He wrote it is so clearly imprinted on his subconscious mind that, even to this day, he can remember it as though it happened recently:

I experienced as though I was being flown away and up by two winged creatures (angels or fairies?) toward the higher skies. Each of them was holding one of my hands and proceeding smoothly up and up! It was an extremely exhilarating journey for my body and soul and I was fully enjoying the same. To my grievous despair, such sublime and joyous moments were being disturbed and broken by interference of some earthly forces that were trying to pull me down by grappling at my feet. In utter disgust I was trying to kick and jerk them down to get rid of their nuisance and continue my upward journey.

He added that he was revived to consciousness by some injections given by the physician who had finally arrived.

This case, which rated a WCEI score of 7 , is especially interesting when compared to one reported by I. R. Judson and E. Wiltshaw (1983). In that case, a 72-year-old man had an initially blissful NDE that was transformed into horror on injection of naloxone, a narcotic antagonist, to bring him out of his coma; it could be that the naloxone disrupted the pleasant experience induced by the man's own endorphins (Blackmore, 1993). It would be most interesting to know what the injections were in this case, but so far I have been unable to find out, as he did not complete the questionnaire.

$N D E$ \#5. A pop star, playing on an outdoor stage on Christmas Day, 1970, was accidentally electrocuted and explained:

I felt "myself" light as a feather, shooting upwards at an indescribable speed-which can never be measured by the words "speed" or "time" and there, below me, above me, surrounding me on all sides were lights of all colours-shining spots which were not moving with me.

After that experience, which rated a WCEI score of 8 , he took up serious study of yoga.

$N D E$ \#6. A 63-year-old man described an experience that happened in 1972 when he had a cardiac arrest while being operated on for a 
liver abscess. He was very ill, with no blood pressure and his breath failing when he felt

some kind of energy trying to leave my body. . . . I could feel my head revolving at an unimaginable speed and I felt that I travelled a few million miles away in an unknown space from the [operating] theatre within a split of a second. I travelled to a space of brilliant light where I was being loved, the love I had never experienced before ... nor am experiencing after that event.

He added

I had a feeling that for hours together I was away from this world enjoying being with the light. Now looking back, at this age of 63 , after having read books, I feel I was beyond time and space. When I opened my eyes around 4:00 p.m., my first words were "Doctor, why did you bring me back? I was very happy where I was."

It was some time before he was able to see again normally. Later he felt that he had developed extrasensory perception and started having mystical experiences. He and his wife now sing devotional songs, bhajans, which he feels helps in his search for spiritual elevation. In the questionnaire, he described a "very bright light, super white with glow"; his experience rated a WCEI score of 17.

To summarize these experiences, out of eight NDErs, one heard sweet music, three reported a tunnel or dark space, three had the sensation of traveling, four saw or experienced light, four had positive emotions of joy or peace, two encountered other beings, and three reported subsequent changes in their lives or beliefs.

\section{Aftereffects}

I have described some of the effects that NDEs had on the people who experienced them. However, it was clear that the people who had no NDE also experienced changes in their lives and beliefs.

A 40 year-old man was knocked down by a car and was unconscious in the hospital for 14 days. He had no NDE and recalled no emotional feelings from that time. His only recollection is that when he felt he was facing death he thought about all the wrong he had done in his life and all the debts he had to clear, and he pleaded with God to let him undo all the wrong. He made a surprising recovery, although he still has to use a wheelchair. He wrote: 
Now, dear Doctor, I must admit to having slipped from the right track
now and then but I've almost stopped sinning. I often, when tempted,
say, "Oh God, deal with me as though [sic] wilt and please assist me
onto the right track."

Two respondents who recalled no experience at all had their faith in God strengthened. One man who was nearly hit by a train claimed that his faith in God became stronger. Another survived a plane crash, escaping before it exploded and leaving his friend behind in the plane. He was left with the thought that he had been saved for a purpose.

Finally, a 60-year-old woman went through a period of great despondency when she thought she might have cancer, and it had a profound and lasting effect on her. She thought very hard about death and life and concluded that it did not matter whether the world or the creatures in it were real or imaginary, as long as one could die and disappear from the world. Likewise, the presence or absence of God became irrelevant. She concluded that the reason people believe in God is to pamper themselves and avoid the fear of death, and at last she realized that everyone is really alone; nothing matters.

Though this realization was deeply depressing at the time, it clearly had longlasting and positive effects. Whatever frightening things she did not want to think about before the incident, afterwards she found she had to, for it might be her last chance. She explained that it "prodded me to 'stand up' and confirm myself." Now, she wrote: "the God I believe in is to try to be 'considerate' to the feelings of others. To me 'right' and 'wrong' have only this as the point of reference."

She also explained that when you accept that at some point you will go into oblivion or nothingness, things like heaven, hell, and God cease to have any meaning. "Then you accept the values of here and now with more honesty, attachment, and as really meaningful. You begin to enjoy living as a 'passing phase' with death being part of it." She added that she was grateful to me for my request, because she felt happy to tell someone about it and had never before told anyone except her brother.

I would like to add here that there is an enduring conflict for people who do not believe in life after death, whether near-death researchers, experiencers, or anyone else. That is, that the idea of a future life seems to give comfort and strength to people who believe in it, and it therefore seems wrong to try to dissuade them from that belief, even if you think it false. This respondent directly addressed this issue when she wrote:

Another very important thing that happens is this: you enjoy the thought of some being ignorant of such things so that they are really 
able to enjoy life in some sort of genuine hopes, that are no more in your case. So you consider it a sin to destroy it by making them know what you think you know.

These reported changes make it possible to address the question of whether it is coming close to death or having an NDE that is responsible for them. Of the seven respondents in this study who had no experiences, 4 (57 percent) reported life changes; of the four who had dreamlike experiences, one ( 25 percent) reported an increase in faith; and of the eight NDErs in this sample, three ( 38 percent) reported life changes.

\section{Discussion}

This study has obvious limitations. The sample was self-selected and is likely to have consisted only of well-educated Indians who speak English well. It is therefore not at all representative of Indians in general.

There were also problems in applying Ring's WCEI to these cases. The accounts with the highest WCEI scores did not seem to be necessarily the deepest or most impressive ones. Ring himself suggested a cutoff score of 6 , below which a person would not count as a "core experiencer." Applying this criterion, the respondent who reported the clearest tunnel, sweet music, and euphoria would not be considered a near-death experiencer. This is particularly interesting because Greyson (1983), in correlating each item of his NDE questionnaire with the whole score, found that tunnel experiences did not help differentiate the depth of the NDE and deleted that item from his final scale. This suggests that the tunnel experience is a separate component from the rest of the NDE.

However, these problems are incidental to the purpose of answering the main question I wished to address here: whether people in India also experience tunnels and lights when coming close to death. The answer was clearly that they do. Three cases (38 percent) included accounts either of tunnels specifically or of dark places. This study is too small and has too many sampling problems to permit reliable comparisons with previous work, but we can make some tentative comments.

For example, Justine Owens, Emily Cook, and Ian Stevenson (1990) found that 21 of 46 NDErs (46 percent) reported being in a tunnel, and Greyson (1983) found 32 percent of NDErs described a "tunnel-like dark region." My figure of 38 percent lies right between these two. 
Also, in his original study of people who had come close to death, Ring (1980) found that 23 percent "entered the darkness," compared to 3 out of 19 (16 percent) in the present study. As for lights, 4 out of 8 among my respondents (50 percent) saw them, compared to 43 percent in Greyson's (1983) study; other studies do not give comparable data.

The aftereffects of coming close to death in this sample are also very similar to those described in previous reports. They include reduced fear of death, increased belief in God, greater concern for others, and living more in the moment. These effects seemed to occur whether or not the person had an NDE. The process of coming close to death and being forced to contemplate its meaning was enough to change these respondents' lives.

The findings of this study clearly differ from Pasricha and Stevenson's (1986), which seemed to show that Indian NDEs were different from the classic type in important respects, including the absence of tunnel experiences. The Indian NDEs reported here include most of the key features of the classic Moody-type NDE and, although this sample is very small, the proportions experiencing tunnels, dark places, and lights are remarkably similar to those in previous studies. In other words, these features seem independent of the person's culture. This conclusion fits with what would be expected if these features are a product of brain physiology and are not dependent on culture.

\section{References}

Blackmore, S. J. (1993). Dying to live: Science and the near-death experience. London, England: Grafton.

Blackmore, S. J., and Troscianko, T. (1989). The physiology of the tunnel. Journal of Near-Death Studies, 8, 15-28.

Carr, D. B. (1982). Pathophysiology of stress-induced limbic lobe dysfunction: A hypothesis relevant for NDEs. Anabiosis: The Journal of Near-Death Studies, 2, 75-89.

Davis, L. (1988). A comparison of UFO and near-death experiences as vehicles for the evolution of human consciousness. Journal of Near-Death Studies, 6, 240-257.

Dobson, M., Tattersfield, A. E., Adler, M. W., and McNicol, M. W. (1971). Attitude and long-term adjustment of patients surviving cardiac arrest. British Medical Journal, 3(5768), 207-212.

Druss, R. G., and Kornfeld, D. S. (1967). The survivors of cardiac arrest: A psychiatric study. JAMA: Journal of the American Medical Association, 201, 291-296.

Flynn, C. P. (1982). Meanings and implications of NDEr transformations: Some preliminary findings and implications. Anabiosis: The Journal of Near-Death Studies, 2, 313.

Gabbard, G. O., and Twemlow, S. W. (1984). With the eyes of the mind: An empirical analysis of out-of-body states. New York, NY: Praeger.

Grey, M. (1985). Return from death: An exploration of the near-death experience. London, England: Arkana. 
Greyson, B. (1983). The Near-Death Experience Scale: Construction, reliability, and validity. Journal of Nervous and Mental Disease, 171, 369-375.

Judson, I. R., and Wiltshaw, E. (1983). A near-death experience. Lancet, 2, 561-562.

Lorimer, D. (1990). Whole in one: The near-death experience and the ethic of interconnectedness. London, England: Arkana.

Moody, R. A., Jr. (1975). Life after life. Covington, GA: Mockingbird.

Morse, M. L., Venecia, D., Jr., and Milstein, J. (1989). Near-death experiences: A neurophysiological explanatory model. Journal of Near-Death Studies, 8, 45-53.

Osis, K., and Haraldsson, E. (1977). Deathbed observations by physicians and nurses: A cross-cultural survey. Journal of the American Society for Psychical Research, 71, 237 259.

Owens, J. E., Cook, E. W., and Stevenson, I. (1990). Features of "near-death experience" in relation to whether or not patients were near death. Lancet, 336, 1175-1177.

Pasricha, S., and Stevenson, I. (1986). Near-death experiences in India: A preliminary report. Journal of Nervous and Mental Disease, 174, 165-170.

Penfield, W. (1955). The role of the temporal cortex in certain psychic phenomena. Journal of Mental Science, 101, 451-465.

Persinger, M. A. (1983). Religious and mystical experiences as artifacts of temporal lobe function: A general hypothesis. Perceptual and Motor Skills, 57, 1255-1262.

Ring, K. (1980). Life at death: A scientific investigation of the near-death experience. New York, NY: Coward, McCann and Geoghegan.

Ring, K. (1984). Heading toward omega: In search of the meaning of the near-death experience. New York, NY: Morrow.

Ring, K. (1992). The Omega Project: Near-death experiences, UFO encounters, and Mind at Large. New York, NY: Morrow.

Saavedra-Aguilar, J., and Gómez-Jeria, J. (1989). A neurobiological model for near-death experiences. Journal of Near-Death Studies, 7, 205-222. 